\title{
Expansiveness, Hyperbolicity and Hausdorff Dimension
}

\author{
Albert Fathi \\ Department of Mathematics, University of Florida, Gainesville, Florida 32611, USA \\ CNRS UA1169, Université Paris-Sud, F-91405 Orsay, France
}

\begin{abstract}
We show that there exists a simple upper bound on the dimension of a hyperbolic compact set of a dynamical system in terms of topological entropy and a uniform contraction rate on the stable and unstable manifolds. This allows us to give proofs of several apparently unrelated theorems.
\end{abstract}

\section{Introduction}

If $f: X \rightarrow X$ is a continuous map on the compact space $X$, we write ent $(f)$ for its topological entropy - see [Wa]. If $\mu$ is a probability measure on $X$ invariant under $f$, we write $h(\mu, f)$ or $h(\mu)$ for the measure theoretic entropy of $f$ with respect to $\mu$-see [Wa].

If $(X, d)$ is a metric space, we will denote by $\operatorname{HD}_{d}(X)$ its Hausdorff dimension - see [F1] or [HW] for the definition. Since our theorems refer in fact to the upper capacity $C_{d}(Y)$-called also the entropy dimension, we are going to recall the definition. Let $(X, d)$ be a metric space, call $N(X, \varepsilon)$ the minimum number of balls of $d$-radius $\varepsilon$ needed to cover $X$, we define:

$$
\bar{C}_{d}(X)=\limsup _{\varepsilon \rightarrow 0} \frac{\log N(X, \varepsilon)}{-\log \varepsilon} .
$$

Recall that $\mathrm{HD}_{d}(X) \leqq \bar{C}_{d}(X)$ and that these two quantities depend only on the Lipschitz class of the metric $d$. If the Lipschitz class of the metric $d$ is clear from the context, we will drop the index $d$; this will be for example the case when $X$ is a subset of a smooth manifold, because in that case the preferred Lipschitz structure will be the one coming from the smooth structure of the manifold.

In this work we show that there exists an easily established inequality between the Hausdorff dimension and the entropy for a hyperbolic compact invariant set. In fact, if $K$ is a compact invariant hyperbolic set for the $C^{1}$-diffeomorphism $f$

1 Partially supported by NSF Grant \# DMS-8801749. Part of this work was done in Spring 87 at The Institute for Advanced Study under NSF Grant \# DMS-8610730(1) 
and $f$ (respectively $f^{-1}$ ) contracts stable (respectively unstable) manifolds by $\rho<1$ then:

$$
\bar{C}(K) \leqq 2 \frac{\operatorname{ent}(f \mid K)}{-\log \rho}
$$

Surprisingly, although the relation given above is straightforward to establish it does not seem to have appeared in print. The most interesting fact about this relation is that it applies in apparently different contexts.

In a first application we show that the Hausdorff dimension of the union of the hyperbolic Aubry-Mather sets in twist diffeomorphisms is 0 . The question of the Hausdorff dimension or even the Lebesgue measure of the union of the Aubry-Mather cantor sets (including the non-hyperbolic ones) was raised several times by Y. Sinai during the London Mathematical Society Meeting on Dynamical Systems in Durham in July 1988. In a different geometrical setting the inequality gives a proof of the Birman-Series theorem which says that simple geodesics in surfaces of negative curvature form a set of Hausdorff dimension one. In the two cases above, the result follows from the fact that we are able to show on general grounds that the topological entropy of the dynamical system restricted to the set is 0 .

The same kind of technique allows us to give a proof of Mañé's theorem which states that a compact set which admits an expansive homeomorphism must have finite topological dimension. This is done by showing that there exists a metric whose Hausdorff dimension is finite; this is essentially a result that was conjectured by David Fried. Another consequence of our methods is that expansive homeomorphisms with zero topological entropy can exist only on compact totally disconnected sets.

We introduce more notations. If $X$ is a manifold $T X$ is its tangent bundle, if $K \subset X$ then $T_{K} X$ is the part of the tangent bundle above $K$, if $x \in X$ the tangent space at $x$ is $T_{x} X$, if $f$ is a $C^{1}$ map $T_{x} f$ is the tangent map at $x$. More generally, we will use the same type of notations for arbitrary fiber bundles.

For convenience, a partial $C^{1}$ flow $f_{t}: U \rightarrow X$, where $U$ is an open subset of the smooth manifold $X$, is a $C^{1}$ map $(x, t) \mapsto f_{t}(x)$ defined on a neighborhood of $U \times 0$ in $U \times \mathbf{R}$ and such that $f_{0}(x)=x$ and $f_{t+t^{\prime}}(x)=f_{t}\left(f_{t^{\prime}}(x)\right)$ whenever this makes sense. Remark that for any compact subset $K$ of $U$ there exists some $\varepsilon>0$ such that $f_{t}(x)$ exists for $x$ in a small neighborhood of $K$ and $|t|<\varepsilon$. So it makes sense to say that $K$ is invariant under $f_{t}$ and in this case one can define $f_{t}$ for all time $t$ on $K$; remark that when $K$ is $f_{t}$ invariant, for $x \in K$, the derivative $T_{x} f_{t}$ is defined for all time $t \in \mathbf{R}$. Recall that a stationary point of $f_{t}$ is a point $x$ that satisfies $f_{t}(x)=x$.

\section{Hausdorff Dimension of Hyperbolic Sets of Diffeomorphisms and Flows}

We recall the notion of hyperbolic invariant set for diffeomorphisms and flows in order to fix notations

Definition 1.1.a) Let $f: U \rightarrow X$ be a $C^{1}$ diffeomorphism of the open subset $U$ of the manifold $X$ onto some open subset of $X$. We say that a compact subset $K \subset U$ is 
hyperbolic for $f$ if it is invariant under $f$ and there exists a $T f$-invariant decomposition $T_{K} X=E^{s} \oplus E^{u}$ such that:

and

$$
\lim _{n \rightarrow+\infty} \frac{1}{n} \log \left(\max _{x \in K}\left\|T_{x} f^{n} \mid E^{s}\right\|\right)<0
$$

$$
\lim _{n \rightarrow+\infty} \frac{1}{n} \log \left(\max _{x \in K}\left\|T_{x} f^{-n} \mid E^{u}\right\|\right)<0 .
$$

Remark that the two quantities above are independent of the choice of a Riemannian metric on $X$-this uses the compactness of $K$. The fact that the limits exist is a consequence of the subadditivity of both sequences $\left(\log \left(\max _{x \in k}\left\|T_{x} f^{n} \mid E^{s}\right\|\right)\right)_{n \in \mid \mathbf{N}}$ and $\left(\log \left(\max _{x \in \mathbf{K}}\left\|T_{x} f^{-n} \mid E^{u}\right\|\right)\right)_{n \in \mid \mathbf{N}}$.

b) Let $f_{t}: U \rightarrow X$ be a partial $C^{1}$ flow defined on the open subset $U$ of the manifold $X$. We say that a compact subset $K \subset U$ is hyperbolic for $f_{t}$ if it is invariant under $f_{t}$, it contains no stationary point and there exists a $T f_{t}$-invariant decomposition $T_{K} X=E^{s} \oplus E^{u} \oplus E^{0}$, where $E^{0}$ is the direction tangent to the flow and:

and

$$
\lim _{t \rightarrow+\infty} \frac{1}{t} \log \left(\max _{x \in \mathbf{K}}\left\|T_{x} f_{t} \mid E^{s}\right\|\right)<0
$$

$$
\lim _{t \rightarrow+\infty} \frac{1}{t} \log \left(\max _{x \in \mathbf{K}}\left\|T_{x} f_{-t} \mid E^{u}\right\|\right)<0 .
$$

Remark that the bundle $E^{0}$ is isomorphic to the trivial bundle $K \times \mathbf{R}$, because $K$ does not contain stationary points.

The proofs of the next two theorems are quite similar, we will prove the second one since it requires more care.

Theorem 1.2. Let $K$ be a compact subset of the manifold $M$. Suppose that $K$ is hyperbolic for the $C^{1}$ diffeomorphism $f$. Define:

$$
\lambda=\max \left[\lim _{n \rightarrow+\infty} \frac{1}{n} \log \left(\max _{x \in K}\left\|T_{x} f^{n} \mid E^{s}\right\|\right), \lim _{n \rightarrow+\infty} \frac{1}{n} \log \left(\max _{x \in K}\left\|T_{x} f^{-n} \mid E^{u}\right\|\right)\right],
$$

then we have:

$$
\mathrm{HD}(K) \leqq \bar{C}(K) \leqq 2 \frac{\operatorname{ent}(f \mid K)}{-\lambda} .
$$

Theorem 1.3. Let $K$ be a compact subset of the manifold $M$. Suppose that $K$ is hyperbolic for the partial $C^{1}$ flow $f_{t}$. Define:

$$
\lambda=\max \left[\lim _{t \rightarrow+\infty} \frac{1}{t} \log \left(\max _{x \in K}\left\|T_{x} f_{t} \mid E^{s}\right\|\right), \lim _{t \rightarrow+\infty} \frac{1}{t} \log \left(\max _{x \in K}\left\|T_{x} f_{-t} \mid E^{u}\right\|\right)\right] .
$$

then we have:

$$
\mathrm{HD}(K) \leqq \bar{C}(K) \leqq 2 \frac{\operatorname{ent}\left(f_{1} \mid K\right)}{-\lambda}+1
$$


Proof. We choose some Riemannian metric on $T_{K}(X)=E^{s} \oplus E^{u} \oplus E^{0}$. We fix some $\varepsilon>0$, subject to the condition $\lambda+\varepsilon<0$. To simplify the notations, we define:

$$
\rho=\exp (\lambda+\varepsilon)<1 \text {. }
$$

Since the conclusion of the theorem does not change if we replace $f_{t}$ by $f_{k t}$ with $k>0$, we can assume that:

$$
\forall x \in K, \quad\left\|T_{x} f_{1}\left|E^{s}\|<\rho, \quad\| T_{x} f_{-1}\right| E^{u}\right\|<\rho .
$$

We can find a smooth map $\theta: \mathcal{O} \rightarrow X$ defined in a neighborhood of the zero section of $T(X)$ above $K$, such that for every $x \in K$ the derivative of $\theta_{x}=\theta \mid \mathcal{O} \cap T_{x}(X)$ at $0_{x}$ is the identity - such a map can be obtained from the exponential map associated to the Riemannian metric on $X$.

Call $E$ the bundle $E^{s} \oplus E^{u} \oplus \mathbf{R}$ over $K$, call $\pi_{s}, \pi_{u}$ and $\pi_{0}$ the projections of the bundle $E$ on $E^{s}, E^{u}$ and $K \times \mathbf{R}$ respectively, we define for $v \in E,\|v\|=$ $\max \left(\left\|\pi_{s}(v)\right\|,\left\|\pi_{u}(v)\right\|,\left\|\pi_{0}(v)\right\|\right)$.

We define a map $\mu: \mathscr{U} \rightarrow X$ on a neighborhood $\mathscr{U}$ of the zero section in the bundle $E=E^{s} \oplus E^{u} \oplus \mathbf{R}$ over $K$ by $\left(v_{s}, v_{u}, t\right) \mapsto f_{t}\left(\theta_{x}\left(v_{s}, v_{u}, 0\right)\right)$ if $\left(v_{s}, v_{u}\right) \in E_{x}^{s} \oplus E_{x}^{u}$. For each $x \in K$ call $\mu_{x}$ the restriction of $\mu$ to $E_{x}$, the family $\mu_{x}, x \in K$ is a $C^{1}$-family, i.e. each $\mu_{x}$ is $C^{1}$ and the derivatives depend continuously on $x \in K$. The derivative at $0_{x}$ is given by $\left(v_{s}, v_{u}, t\right) \mapsto\left(v_{s}, v_{u}, t V(x)\right)$, where $V(x)=\left.\left(d f_{t}(x) / d t\right)\right|_{t=0}$. This implies that $\mu_{x}$ is a $C^{1}$ diffeomorphism of some open neighborhood of $0_{x}$ in $E_{x}$ onto some open neighborhood of $x \in X$.

For each $x \in K$, we define $\tilde{f}_{x}=\mu_{f_{1}(x)}^{-1} f_{1} \mu_{x}$. Due to the compactness of $K$, there exists some $r>0$ such that for every $x \in K$, the open $C^{1}$ embedding $\tilde{f}_{x}$ is defined on $B\left(0_{x}, r\right)$, the ball of center zero and radius $r$ in $E_{x}$ and maps into $E_{f_{1}(x)}$. Moreover, it has the form:

$$
\left(v_{s}, v_{u}, t\right) \mapsto\left(F_{1}\left(v_{s}, v_{\mu}\right), F_{2}\left(v_{s}, v_{u}\right), t+\varphi\left(v_{s}, v_{u}\right)\right) .
$$

This follows from the fact that $\mu_{x}$ takes the $\mathbf{R}$ factor of the fiber $E_{x}$ onto trajectories of the flow $f_{t}$. For each $x \in K$, the derivative of $\tilde{f}_{x} \mid E^{s} \oplus E^{u}$ at $0_{x}$ is equal to $T_{x} f_{1} \mid E^{s} \oplus E^{u}$. It follows from (2) and (3) that there exists some $\alpha_{0}>0$ such that:

$$
\begin{aligned}
\forall x \in K, \quad \forall v & =\left(v_{s}, v_{u}, t\right) \in B\left(0_{x}, \alpha_{0}\right), \\
\left\|\pi_{s}\left(\tilde{f}_{x}(v)\right)\right\| & \leqq \rho \max \left(\left\|v_{s}\right\|,\left\|v_{u}\right\|\right), \\
\left\|\pi_{u}\left(\tilde{f}_{x}^{-1}(v)\right)\right\| & \leqq \rho \max \left(\left\|v_{s}\right\|,\left\|v_{u}\right\|\right) .
\end{aligned}
$$

We define $P_{x}(a, b, c)=\left\{v=\left(v_{s}, v_{u}, t\right) \in E_{x}\left|\left\|v_{s}\right\| \leqq a,\left\|v_{u}\right\| \leqq b,\right| t \mid \leqq c\right\}$ - of course, we have $P_{x}(a, a, a)=B\left(0_{x}, a\right)$. Since $\left(\mu_{x}\right)_{x \in K}$ is a $C^{1}$ family and $K$ is compact, by making $\alpha_{0}$ smaller if necessary, we can find $C>0$ such that:

$$
\begin{gathered}
\forall x \in K, \quad \forall a<\alpha_{0}, \\
B\left(x, C^{-1} a\right) \subset \mu_{x}\left(P_{x}(a, a, a)\right) \subset B(x, C a),
\end{gathered}
$$

where $B(x, r)$ is the ball of radius $r$ in $x$ for the Riemannian metric that we fixed in the beginning. Since for $x \in K$ and $b \geqq a$ the set $P_{x}(a, a, b)$ is contained in the union of $(b / a)+1$ balls of radius $a$ in $E_{x}$, by making $C$ larger, if necessary, we can 
assume that:

$$
\forall x \in K, \quad \forall 0<a \leqq b<\alpha_{0},
$$

$\mu_{x}\left(P_{x}(a, a, b)\right) \subset$ in the union of at most $(b / a)+1$ balls of radius $C a$ in $X$.

For $x \in K, n \in \mathbf{N}$ and $\alpha \leqq \alpha_{0}$ where denote by $B_{n}\left(0_{x}, \alpha\right)$ the set of $v \in E_{x}$ such that $\|v\| \leqq \alpha$ and for $i=1, \ldots, n,\left\|\tilde{f}_{f_{i-1}(x)} \cdots \tilde{f}_{x}(v)\right\| \leqq \alpha$ and $\left\|\tilde{f}_{f_{-(i-1)(x)}^{-1}} \cdots \tilde{f}_{x}^{-1}(v)\right\| \leqq \alpha$. From (3) we have:

$$
\forall x \in K, \quad \forall n \in \mathbf{N}, \quad B_{n}\left(0_{x}, \alpha\right) \subset P_{x}\left(\rho^{n} \alpha, \rho^{n} \alpha, \alpha\right) .
$$

Let us call $N(n, \alpha)$ the minimum number of sets of the form $\mu_{x}\left(B_{x}(n, \alpha)\right)$ needed to cover $K$. By (5) and the definitions, for $x \in K$ a set $\mu_{x}\left(B_{x}(n, \alpha)\right)$ contains $\bigcap_{i=n}^{i=n} f_{1}^{-i}\left[B\left(f_{1}^{i}(x), C^{-1} \alpha\right) \cap K\right]$. If we take images by $f^{-n}$, we see that $N(n, \alpha)$ is smaller than the minimum cardinality of an $\left(2 n+1, C^{-1} \alpha\right)$-spanning set for $f_{1} \mid K$-see [Wa, $\$ 7.2$ page 168$]$ for the definition of a spanning set. It follows that:

$$
\lim _{\alpha \rightarrow 0} \limsup _{n \rightarrow \infty} \frac{\log N(n, \alpha)}{n} \leqq 2 \operatorname{ent}\left(f_{1} \mid K\right) \text {. }
$$

from (6) and (7):

$$
\forall \alpha<\alpha_{0}, \quad \bar{C}(K) \leqq \limsup _{n \rightarrow \infty} \frac{\log \left(N(n, \alpha)\left[\rho^{-n}+1\right]\right)}{-\log \left(C \rho^{n} \alpha\right)} \leqq \frac{\limsup \frac{\log N(n, \alpha)}{n}}{-\log \rho}+1
$$

(The first inequality above follows, for example from [Ft, Lemma 6.2, page 197].) From (1) $\log \rho=\lambda+\varepsilon$. The theorem follows from (8) and (9) by letting $\alpha$ and $\varepsilon$ tend to 0 .

Remark 1.4. The preceding theorem is very close to [Ft, Theorem 6.3]. The advantage here is that we do not use the existence of the stable and unstable manifolds. The first proof we found of Theorem 1.2 was using the stable and unstable manifolds and the local product structure defined by the two foliations but that introduces a constant which is the Hölder exponent relating the Lipschitz structure obtained from the smooth structure. Of course, here like in [Ft] we are using the main observation of [DO], which is how to compute capacity and Hausdorff dimension with parallelotopes instead of cubes. Using the same ideas, it is possible to find a better estimate than the one above by using uniform Lyapunov exponents.

Corollary 1.5. Let $f: U \rightarrow X$ be a $C^{1}$ diffeomorphism of the open subset $U$ of the manifold $X$ into $X$. Suppose $K$ is a compact subset of $U$ which is invariant and hyperbolic under $f$. If $\operatorname{ent}(f \mid K)=0$, then we have:

$$
\mathrm{HD}(K)=\bar{C}(K)=0 \text {. }
$$

Corollary 1.6. Let $f_{t}: U \rightarrow X$ be a partial $C^{1}$ flow defined on the open subset $U$ of the manifold $X$ into $X$. Suppose $K$ is a compact subset of $U$ which is hyperbolic for $f_{t}$. If $K$ is not empty and ent $\left(f_{1} \mid K\right)=0$, then we have:

$$
\mathrm{HD}(K)=\bar{C}(K)=1 \text {. }
$$


Proof. Remark that $\mathrm{HD}(K) \geqq 1$ because $K$ contains at least one orbit of the flow and there are no stationary points of $f_{t}$ in $K$.

\section{Dimension of Hyperbolic Aurbry-Mather Sets}

Denote by $A$ the annulus $(\mathbf{R} / \mathbf{Z}) \times \mathbf{R}$. Call $\pi$ the projection of $A$ onto the first factor $\mathbf{R} / \mathbf{Z}$. A twist $C^{1}$ diffeomorphism of $A$ is a diffeomorphism $f(x, y)=\left(f_{1}(x, y), f_{2}(x, y)\right)$ isotopic to the identity and such that there exists $k>0$ with $\left(\partial f_{1} / \partial y\right)(x, y)>k$ for all $(x, y) \in A$. See $[\mathrm{AL}],[\mathrm{Ch}],[\mathrm{Ka}]$ and $[\mathrm{Mt}]$ for facts on twist diffeomorphisms.

An ordered orbit for $f$ is an orbit which projects under $\pi$ injectively into $\mathbf{R} / \mathbf{Z}$ and such that the bijection obtained from $f$ on the projection extends to a homeomorphism of the circle $\mathbf{R} / \mathbf{Z}$. From [Ka, Corollary 1 page 188 ], it follows that the closure of an ordered orbit consists or ordered orbits, moreover, the union of the ordered orbits is a closed set. Aubry-Mather theory shows that conservative twist diffeomorphisms which admit a Hamiltonian have a large set of ordered orbits. For the sake of simplicity, let us say that an ordered orbit is hyperbolic if its closure is hyperbolic in the usual sense. Remark that the set of ordered hyperbolic orbits is in general not closed but is anyway a countable union of compact hyperbolic sets with each one of them a union of ordered orbits-see the proof 2.1 below.

In [MK], Robert MacKay showed that an Aubry-Mather hyperbolic Cantor set has upper capacity $0^{2}$. The result of MacKay is an improvement of [Ka, Proposition 4 page 193] in the (uniformly) hyperbolic case (the reader should notice that in $[\mathrm{Ka}]$ the word hyperbolic means that there is no zero Lyapunov exponent). Our goal is to give a simple proof of the following fact, which generalizes MacKay result:

Theorem 2.1. In a twist $C^{1}$ diffeomorphism $f$ of the annulus the union of the hyperbolic ordered orbits has Hausdorff dimension 0. Moreover, any compact subset of that set has also upper capacity 0.

Proof. Fix some Riemmanian metric on the annulus $A$, call $\|v\|$ the norm of a tangent vector for that Riemannian metric. For each $i \in \mathbf{N}$, we say that a compact set $K$ invariant under $f$ satisfy the condition $P_{i}$ if $K \subset(\mathbf{R} / \mathbf{Z}) \times[-i, i]$ and there exists a decomposition $T_{K} A=E^{s} \oplus E^{u}$ into two $T f$-invariant subbundles such that:

$$
\begin{gathered}
\forall v \in E^{s}, \quad \forall n \geqq 1, \quad\left\|T f^{n}(v)\right\| \leqq i\left(1-\frac{1}{i}\right)^{n}\|v\|, \\
\forall w \in E^{u}, \quad \forall n \geqq 1, \quad\left\|T f^{-n}(w)\right\| \leqq i\left(1-\frac{1}{i}\right)^{n}\|w\| .
\end{gathered}
$$

It is well known that the union $K_{i}$ of the sets satisfying $P_{i}$ is compact and satisfies also $P_{i}$. Of course, any compact invariant hyperbolic subset is contained in $K_{i}$ for

\footnotetext{
2 Notice that the second argument in that note, deducing the result from L. S. Young work, is not correct
} 
all large enough $i$. Moreover, from [Ka, Corollary 1 page 188], it is clear that $L_{i}$ the intersection of $K_{i}$ with the set of ordered orbits is a compact invariant hyperbolic subset for $f$. Also any compact subset of the union of hyperbolic ordered orbits is contained in $L_{i}$ for all large $i$ and the union of all hyperbolic ordered orbits sets is the union of the $\mathrm{L}_{i}$ 's.

To prove the theorem, using 1.5, we have to show that the topological entropy ent $\left(f \mid L_{i}\right)$ is zero. Since the topological entropy of a map is the supremum of its measure theoretic entropy for ergodic invariant measures-see [Wa, Corollary 8.6.1 page 190], it suffices to prove that the metric theoretic of an $f$-invariant ergodic measure supported by $L_{i}$ is 0 . Such an $f$-invariant ergodic measure $\mu$ supported by $L_{i}$ is in fact supported by the closure of an ordered orbit. By the definition of ordered orbit, we are, in fact, trying to compute metric theoretic for an invariant measure of a homeomorphism of the circle, it follows that the measure theoretic entropy of $f$ for $\mu$ is 0 .

An ordered orbit of a twist diffeomorphism comes with a rotation number in $\mathbf{R} / \mathbf{Z}$, namely the rotation number of the homeomorphism of $\mathbf{R} / \mathbf{Z}$ that is obtained by extending the map induced by the diffeomorphism on the projection of the orbit under $\pi$.

Theorem 2.2. For a generic conservative twist $C^{1}$ diffeomorphism of the annulus which admits a Hamiltonian, the map from the union of the ordered orbits to $\mathbf{R} / \mathbf{Z}$ which maps a point on the rotation number of its orbit is not Hölder.

Proof. We will have to work in the universal cover $\tilde{A}$ of the annulus $A$. From [LC], for a generic conservative twist $C^{\mathbf{1}}$ diffeomorphism there is an open non-empty interval $I$ in $\mathbf{R}$ such that any ordered orbit in $\tilde{A}$ whose rotation number is in $I$ is hyperbolic. Since by Aubry-Mather theory we get any rotation number in the conservative case, we find that the map which takes a point of an ordered orbit onto the rotation number sends a set of Hausdorff dimension zero on an interval which has Hausdorff dimension 1, hence it cannot be Hölder.

Remark. a) An Aubry-Mather set is a minimal set which is the closure of an ordered orbit - hence it is itself a union of ordered orbits. In particular, the union of the hyperbolic Aubry-Mather sets has Hausdorff dimension 0.

b) There are cases where all ordered orbits are hyperbolic - see [Go] or [He].

\section{Geodesic Laminations and Entropy}

Our goal is to give a dynamical system proof $^{3}$ of the following result - see [BS]:

Theorem 3.1. (Birman and Series). Let $M$ be a surface on which we have a Riemannian metric of strictly negative curvature. Call $S(M)$ the unit tangent bundle and call $p: S(M) \rightarrow M$ the canonical projection. A geodesic in $M$ is called simple if it is defined for all time and it has no transverse self intersection. Call $\hat{\mathscr{S}}$ the subset of $S(M)$ consisting of vectors tangent to some simple geodesic. Any non-empty compact

3 This proof is also known to W. Thurston 
subset of $\hat{\mathscr{S}}$, invariant under the geodesic flow, has Hausdorff dimension 1. In the case where $M$ is compact then $\hat{\mathscr{S}}$ itself is a compact set of upper capacity and Hausdorff dimension equal to one.

The proof is very analogus to the one of Theorem 2.1 above. We do not claim that it is either shorter or easier than the original proof of Birman and Seires, it is just a different perspective on the result.

We will need the concept of geodesic lamination see either [BC] or [Th].

Lemma 3.2. Let $M$ be a surface on which we have a Riemannian metric of strictly negative curvature. Suppose that $\mathscr{G}$ is a compact subset of $\hat{\mathscr{S}}$ which contains a geodesic dense in $\mathscr{G}$, then $p \mid \mathscr{G}$ is at most two to one, moreover the image $p(\mathscr{G})$ is a compact geodesic lamination.

Proof. Call $g$ a geodesic whose lift to $S(M)$ is contained in $\mathscr{G}$ and is dense in $\mathscr{G}$. Since $\mathscr{G}$ is contained in $\hat{\mathscr{S}}$, the closure of $g$ in $M$ is a geodesic lamination. Moreover, the closure of $g$ is precisely $p(\mathscr{G})$. The fact that $p \mid \mathscr{G}$ is at most two to one follows from the fact that above a point in a geodesic lamination there is exactly two unit vectors tangent to that lamination (of course, they are opposite vectors).

Lemma 3.3. (L. S. Young). Suppose that $\mathscr{F}$ is a goedesic lamination in a surface $N$, then the topological entropy of any flow defined on $\mathscr{F}$ is zero.

Proof. (Sketch). We use the arguments of [Yo1]. Take enough closed disjoint simple geodesics not contained in $\mathscr{F}$ to obtain the flow as a suspension. Since the return map on the union of these closed geodesics is a piecewise monotone bijection, we can apply [Yo1, Lemma 1, page 469].

Corollary 3.4. Let $M$ be a surface on which we have a Riemannian metric of strictly negative curvature. Suppose that $K \subset \hat{\mathscr{S}}$ is a compact subset which is invariant under the geodesic flow, then the topological entropy of the geodesic flow restricted to $K$ is 0 .

Proof. Any ergodic invariant measure of the geodesic flow restricted to $K$ is carried by a compact invariant set $\mathscr{G}$ which contains a dense orbit. From 3.2, we can assume, by taking if necessary a ramified cover of our surface, that $\mathscr{G}$ is a geodesic lamination sitting in a surface. From 3.3, it follows that the measure theoretic entropy of the ergodic measure is zero. Of course, since the topological entropy is the supremum of the measure ergodic entropy of ergodic invariant measures, we obtain that the topological entropy is also 0 .

Proof of Theorem 3.1. We use that any such set $K$ is, in fact, hyperbolic for the geodesic flow. Moreover, by 3.4, the topological entropy of the geodesic flow restricted to $K$ is 0 . We conclude by 1.6 that the upper capacity and the Hausdorff dimension of $K$ are both 1. To prove the last part of the theorem we have to remark that $\hat{\mathscr{S}}$ is always closed because its complement is defined by an open condition - namely, to have at least one transversal intersection.

Remark 3.5. If we define $\hat{\mathscr{S}}_{k}$ as the set of geodesics whose projection on the surface have at most $k$-transversal intersections then we can use the same proof to get the corresponding theorem. The only thing that we have to remark is that if a geodesic $g$ is in $\widehat{S}_{k}$ and is not closed then its $\omega$-limit set is a geodesic lamination, 
because any transversal intersection in the $\omega$-limit set would force $g$ to have infinitely many intersections.

\section{Hausdorff Dimension of Compact Invariant Subsets of Geodesic Flows of Riemannian Manifolds of Constant Negative Curvature}

Theorem 4.1. Let $M$ be a Riemannian manifold of constant negative curvature $-k^{2}$. Let $K$ be a non-empty subset of the unit tangent bundle $s(M)$. If $K$ is invariant under the geodesic flow, call $h_{K}$ the topological entropy of the geodesic flow restricted to $K$. Then we have:

$$
\mathrm{HD}(K)=\bar{C}(K)=\frac{2 h_{K}}{k}+1
$$

Proof. All the positive (respectively negative) Lyapunov exponents of the geodesic flow are uniform in the direction transverse to the flow and have $k$ (respectively $-k)$ as their common value. The inequality $\bar{C}(K) \leqq\left(2 h_{K} / k\right)+1$ follows from Theorem 1.3. We will show that the other inequality $\operatorname{HD}(K) \geqq\left(2 h_{K} / k\right)+1$ follows from [LY] or [Yo2]. In fact if $\mu$ is any probability measure on $S(M)$ invariant under the geodesic flow, we have from the flow version of [Yo2, Corollary 5.1, page 122]:

$$
\mathrm{HD}(\mu)=\frac{2 h(\mu)}{k}+1
$$

If $\mu(K)=1$ then of course $\operatorname{HD}(\mu) \leqq \operatorname{HD}(K)$. Moreover, we have:

$$
\sup \{h(\mu) \mid \mu(K)=1\}=h_{K} .
$$

\section{Expansive Sets are Hyperbolic. Consequences}

A homeomorphism $h: C \rightarrow C$ of the compact metric space $C$ is expansive, if there exists $\varepsilon>0$ such that:

$$
\forall x \neq y \in C, \quad \sup _{n \in \mathbf{Z}} d\left(h^{n}(x), h^{n}(y)\right)>\varepsilon,
$$

where $d$ is any metric defining the topology of $C$.

After we finished writing down this section, Sheldon Newhouse brought to our attention the work of David Fried [Fe2]. In this paper, David Fried uses Frink's metrization theorem to find a metric which is contracted on the stable sets and dilated on the unstable sets of an expansive map. What we prove in 5.1 is that this metric verifies a stronger inequality than the one you would expect if you do not have a local product structure. It is this stronger inequality that allows us to prove Corollary 5.5, in order to give a proof of Mañé's Theorem 5.6. Other previous instances of use of Frink's metrization theorem in dynamics are due to Coven, Reddy and Fried - see [CR, Re and Fe1]. We learned Frink's theorem in the form we are using it from Patrice Assouad; we are most grateful to him for that knowledge. 
Theorem 5.1. (Expansive homeomorphisms are hyperbolic). If $h: C \rightarrow C$ is an expansive homeomorphism of the compact metric space $C$, then there exists a metric $d$ on $C$ defininig its topology and numbers $k>1, \varepsilon>0$ such that:

$$
\forall x, y \in C, \quad \max \left(d(h(x), h(y)), d\left(h^{-1}(x), h^{-1}(y)\right)\right) \geqq \min (k d(x, y), \varepsilon) .
$$

Moreover, both $h$ and $h^{-1}$ are Lipschitz for $d$.

Proof. Choose some metric $\delta$ on $C$ defining its topology. Call $\varepsilon$ some expansive constant for $h$, i.e.:

$$
\forall x \neq y \in C, \quad \sup _{n \in \mathbf{Z}} \delta\left(h^{n}(x), h^{n}(y)\right)>\varepsilon .
$$

For $x, y \in C$, define $n(x, y)$ by:

$$
n(x, y)= \begin{cases}\infty, & \text { if } x=y \\ \min \left\{n_{0} \in \mathbf{N} \mid \max _{|n| \leqq n_{0}} \delta\left(h^{n}(x), h^{n}(y)\right)>\varepsilon\right\} & \text { if } x \neq y .\end{cases}
$$

Fix some $\alpha>1$, and define a function $\rho: C \times C \rightarrow \mathbf{R}_{+}$by $\rho(x, y)=\alpha^{-n(x, y)}$. Clearly $\rho(x, y)=\rho(y, x)$ and $\rho(x, y)=0$ if and only if $x=y$. Moreover, although it is not a metric, the function $\rho$ defines the topology of $C$, because $h$ is an expansive homeomorphism.

We have:

If

then

$$
\max _{|i| \leqq n-1} \rho\left(h^{i}(x), h^{i}(y)\right) \leqq \frac{1}{\alpha},
$$

$$
\max \left(\rho\left(h^{n}(x), h^{n}(y)\right), \rho\left(h^{-n}(x), h^{-n}(y)\right)\right) \geqq \alpha^{n} \rho(x, y) .
$$

We will now make precise the choice of $\alpha$. We can find an integer $m$ such that $\delta(a, b)>\varepsilon / 2$ implies $n(a, b) \leqq m$. Now choose $\alpha$ such that $\alpha^{m} \leqq 2$. Let $x, y \in C$, if $z \in C$, then either $n(z, y) \leqq m+n(x, y)$ or $n(z, x) \leqq m+n(x, y)$ - this follows from the triangular inequality for the metric $\delta$ and the definition of $m$. We obtain the following inequality:

$$
\rho(x, y) \leqq 2 \max (\rho(x, z), \rho(z, y)) .
$$

We are now in the position to apply Frink's metrization theorem - see [Fn, page 135]. There exists a metric $D$ on $C$ such that:

$$
D(x, y) \leqq \rho(x, y) \leqq 4 D(x, y) .
$$

This implies that $D$ defines the topology on $C$. From (2) and (4), we get

If $\max _{|i| \leqq n-1} D\left(h^{i}(x), h^{i}(y)\right) \leqq \frac{1}{4 \alpha}$,

then $\max \left(D\left(h^{n}(x), h^{n}(y)\right), D\left(h^{-n}(x), h^{-n}(y)\right)\right) \geqq \frac{\alpha^{n}}{4} D(x, y)$. 
Choose $n_{0}$ such that $K=\left(\alpha^{n_{0}} / 4\right)>1$. Let $k=K^{1 / n_{0}}$. Using the Mather trick, we define a metric $d$ by:

$$
d(x, y)=\max _{|i| \leqq n_{0}-1} \frac{D\left(h^{i}(x), h^{i}(y)\right)}{k^{i}}
$$

Of course, the metric $d$ defines the topology of $C$.

By direct inspection, it is easy to establish the following inequality:

$$
\max \left[d(h(x), h(y)), d\left(h^{-1}(x), h^{-1}(y)\right)\right] \geqq \max _{0<|i| \leqq n_{0}} \frac{D\left(h^{i}(x), h^{i}(y)\right)}{k^{|i|-1}} .
$$

Now this last quantity is the maximum of the following two quantities $A$ and $B$ :

$$
A=\max _{0<|i|<m_{0}} \frac{D\left(h^{i}(x), h^{i}(y)\right)}{k^{|i|-1}}=k \max _{0<|i|<n_{0}} \frac{D\left(h^{i}(x), h^{i}(y)\right)}{k^{|i|}}
$$

and

$$
B=\frac{\max \left(D\left(h^{n_{0}}(x), h^{n_{0}}(y)\right), D\left(h^{-n_{0}}(x), h^{-n_{0}}(y)\right)\right)}{k^{n_{0}}} .
$$

Suppose now that $d(x, y)<\frac{1}{4 \alpha k^{n_{0}-1}}$, then by (5) (6) and the definition of $k$, we get:

$$
B \geqq k D(x, y) .
$$

It is easy to conclude from (8) and (10) that we have:

$$
\text { If } d(x, y)<\frac{1}{4 \alpha k^{n_{0}-1}}
$$

then $\max \left(d(h(x), h(y)), d\left(h^{-1}(x), h^{-1}(y)\right)\right) \geqq k d(x, y)$.

Since $C$ is compact, we can find $\varepsilon>0$ such that:

$$
\text { If } d(x, y) \geqq \frac{1}{4 \alpha k^{n_{0}-1}}
$$

then $\max \left(d(h(x), h(y)), d\left(h^{-1}(x), h^{-1}(y)\right)\right) \geqq \varepsilon$.

From (11) and (12), we obtain:

$$
\forall x, y \in C, \quad \max \left(d(h(x), h(y)), d\left(h^{-1}(x), h^{-1}(y)\right)\right) \geqq \min (k d(x, y), \varepsilon) .
$$

To prove that $h$ and $h^{-1}$ are Lipschitz, we remark that they are Lipschitz with Lipschitz constant $\alpha$ for $\rho$; after that all the metrics we defined were in the same Lipschitz class as $\rho$.

Definition 5.2. We propose to call a metric like the one in 5.1 an adapted hyperbolic metric. Remark also that any homeomorphism that admits a hyperbolic metric is expansive-see 5.3 below. 
Theorem 5.3. ${ }^{4}$ Let $h: X \rightarrow X$ be a homeomorphism of the compact metrizable space $X$. Suppose that there exists a metric $d$ on $X$ defining its topology and numbers $k>1, \varepsilon>0$ verifying:

$$
\forall x, y \in X, \quad \max \left(d(h(x), h(y)), d\left(h^{-1}(x), h^{-1}(y)\right)\right) \geqq \min (k d(x, y), \varepsilon) .
$$

Then $h$ is expansive and we have:

$$
\mathrm{HD}_{d}(X) \leqq \bar{C}_{d}(X) \leqq 2 \frac{\operatorname{ent}(h)}{\log k} .
$$

In particular, the upper capacity $\bar{C}_{d}(X)$ and the Hausdorff dimension $\mathrm{HD}_{d}(X)$ are finite.

Proof. An induction on $n$ gives:

If

then

$$
\max _{|i|<n} d\left(h^{i}(x), h^{i}(y)\right)<\frac{\varepsilon}{k}
$$

$$
\max \left(d\left(h^{n}(x), h^{n}(y)\right), d\left(h^{-n}(x), h^{-n}(y)\right)\right)=\max _{|i| \geqq n} d\left(h^{i}(x), h^{i}(y)\right) \geqq k^{n} d(x, y) .
$$

This shows that $h$ is expansive. Moreover, for all $\alpha<(\varepsilon / k)$, the set $B_{n}(x, \alpha)=\left\{y \mid \max _{|i| \leqq n} d\left(h^{i}(x), h^{i}(y)\right) \leqq \alpha\right\}$ is contained in the ball of center $x$ and radius $k^{-n} \alpha$. On the other hand, if we call $N(n, \alpha)$ the minimum number of sets of the form $B_{n}(x, \alpha)$ needed to cover $X$, then, as in 1.3 , we have:

$$
\lim _{\alpha \rightarrow 0} \limsup _{n \rightarrow \infty} \frac{\log N(n, \alpha)}{n} \leqq 2 \operatorname{ent}(h) .
$$

As in 1.3 , it follows that:

$$
\mathrm{HD}_{d}(X) \leqq \bar{C}_{d}(X) \leqq 2 \frac{\text { ent }(h)}{\log k} .
$$

The last part of the statement follows from the fact that the topological entropy of an expansive homeomorphism is finite-see [Wa, Corollary 7.11.1, page 177].

Corollary 5.4. (Mañé). If a compact metric space admits an expansive homeomorphism then its topological dimension is finite.

Proof. From 5.1 and 5.3, there exists a metric defining the topology of the space, for which it has finite Hausdorff dimension. But the topological dimension of a compact space is less than it has finite Hausdorff dimension for any metric defining its topology — see [HW, Sect. 4, page 107].

4 Taken together with Theorem 5.1, this corollary proves a conjecture of David Fried [Fe2, page 506, end of Remark 4] 
Corollary 5.5. If a compact metric space admits an expansive homeomorphism whose topological entropy is 0 then its topological dimension is zero.

Proof. This is a consequence of 5.1 and 5.3.

To complete the picture let us recall that Kushnirenko's theorem $[\mathrm{Ku}]$ can be given the following form, see [It] and [St]:

Theorem 5.6. Let $(X, d)$ be a compact metric space. Its lower capacity $\underline{C}_{d}(X)$ is defined by:

$$
\underline{C}_{d}(X)=\liminf _{\varepsilon \rightarrow 0} \frac{N(X, \varepsilon)}{-\log \varepsilon}
$$

where as before $N(X, \varepsilon)$ is the minimum number of balls of radius $\varepsilon$ needed to cover $X$. Of course $\underline{C}_{d}(X) \leqq \bar{C}_{d}(X)$.

Let $h: X \rightarrow X$ be a continuous map. Define:

$$
\operatorname{Liploc}_{d}(h)=\lim _{\epsilon \rightarrow 0} \sup _{d(x, y)<\varepsilon} \frac{d(h(x), h(y))}{d(x, y)} .
$$

If $\operatorname{Liploc}_{d}(h)<\infty$ and $\underline{C}_{d}(X)<\infty$, then:

$$
\operatorname{ent}(h) \leqq \underline{C}_{d}(X) \max \left(\log \operatorname{Liploc}_{d}(h), 0\right) .
$$

\section{References}

[AL] Aubry, S., Le Daeron, P. Y.: The discrete Frenkel-Kantorova model and its extensions. Physica 8D, 381-422 (1983)

[BS] Birman, J., Series, C.: Geodesics with bounded intersection number on a surface are sparsely distributed. Topology 24, 217-225 (1985)

[BC] Bleiler, S., Casson, A.: Automorphisms of surfaces after Nielsen and Thurston Cambridge: Cambridge University Press 1988

[Ch] Chenciner, A.: La dynamique au voisinage d'un point fixe elliptique conservatif: De Poincaré et Birkhoff à Aubry et Mather. Séminaire Bourbaki Exposé 622. Astérisque 121-122, 147-170 (1985)

[CV] Coven, E., Reddy, W.: Positively expansive maps of compact manifolds. In: Global theory of dynamical systems, Lecture notes in Mathematics. pp. 96-110, New York, Heidelberg, Berlin: Springer 1980

[DO] Douady, A., Oesterlé, J.: Dimension de Hausdorff des attracteurs. C. R. Acad. Sci. Paris Sér. I Math. 290, 1135-1138 (1980)

[Fl] Falconer, K.: The geometry of fractal sets. Cambridge: Cambridge University Press 1985

[Ft] Fathi, A.: Some compact invariant subsets for hyperbolic linear automorphisms of torii. Ergod. Th. Dynam. Syst. 8, 191-204 (1988)

[Fe1] Fried, D.: Métriques naturelles sur les espaces de Smale. C. R. Acad. Sc. Paris 297, 77-79 (1983)

[Fe2] Fried, D.: Finitely presented dynamical systems. Ergod. Th. Dynam. Syst. 7, 489-507 (1987)

[Fn] Frink, A.: Distance functions and the metrization problem. Bull. Am. Math. Soc. 43, 133-142 (1937)

[Go] Goroff, D.: Hyperbolic sets for twist maps. Ergod. Th. Dynam. Syst. 5, 337-339 (1985)

[He] Herman, M.: Remarques sur les Cantors de Mather et Aubry. Conférence à l'École Polytechnique le 14 février 1983

[HW] Hurewicz, W., Wallman, H.: Dimension theory. Princeton, NJ: Princeton University Press 1941

[It] Ito, S.: An estimate from above for the entropy and the topological entropy of a $C^{1}$-diffeomorphism. Proc. Jpn. Acad. 46, 226-230 (1970) 
[Ka] Katok, A.: Some remarks on Birkhoff and Mather twist map theorems. Ergod. Th. Dynam. Syst. 2, 185-194 (1982)

[Ku] Kushnirenko, G.: Upper bound of the entropy of a classical dynamical system. Dokl. Akad. Nauk. SSSR 22, 57-59 (1967)

[LC] Le Calvez, P.: Les ensembles d'Aubry-Mather d'un difféomorphisme conservatif de l'anneau déviant la verticale sont en général hyperboliques. C. R. Acad. Sci. Paris Sér. I Math. 306, 51-54 (1988)

[LY] Ledrappier, F., Young, L. S.: The metric entropy of diffeomorphisms part II: Relations between entropy, exponents and dimension. Ann. Math. 122, 540-574 (1985)

[MK] MacKay, R.: Hyperbolic Cantori have dimension zero. J. Phys. 20A, L559-561 (1987)

[Mn] Mañé, R.: Expansive homeomorphisms. Trans. Am. Math. Soc. 79, 312-319 (1979)

[Mt] Mather, J.: Existence of quasi-periodic orbits for twist homeomorphisms of the annulus. Topology 21, 457-467 (1982)

[Re] Reddy, W. L.: Expansive canonical coordinates are hyperbolic. Topology and its Applications 15, 205-210 (1983)

[St] Strelcyn, J. M.: Leçons sur quelques notions élémentaires de la Théorie ergodique. Istituto di Fisica dell'Università di Parma, Parma, 1975

[Th] Thurston, W: The geometry and topology of 3-manifolds. Lecture notes, Princeton University

[Wa] Walters, P.: An introduction to ergodic theory. Berlin, Heidelberg, New York: Springer 1982

[Yo1] Young, L. S.: Entropy of continuous flows on compact 2-manifolds. Topology 16, 469-471 (1977)

[Yo2] Young, L. S.: Dimension, entropy and Lyapunov exponents. Ergod. Th. Dynam. Syst. 2, 109-124 (1982)

Communicated by J. N. Mather

Received December 30, 1988; in revised from June 1, 1989 